\title{
Narrative Expositionstherapie bei einem Flüchtling mit schwerer PTBS in der stationären Regelversorgung
}

\author{
Narrative Exposure Therapy in a Refugee with Severe PTSD \\ within the Inpatient Standard Care
}

\author{
Autoren \\ Dana Bichescu-Burian, Stefan Tschöke, Raoul Borbé \\ Institut \\ Klinik für Psychiatrie und Psychotherapie I der Universität \\ Ulm, Zentrum für Psychiatrie Südwürttemberg, \\ Ravensburg-Weissenau

\section{Schlüsselwörter} \\ Flüchtlinge, PTBS, narrative Expositionstherapie, stationäre \\ Regelversorgung, psychosoziale Integration
}

Keywords

refugees, PTSD, narrative exposure therapy, inpatient

standard care, psychosocial care

Bibliografie

DOI https://doi.org/10.1055/a-0726-0409

Online-Publikation: 31.10.2018 | Psychiat Prax 2019; 46:

106-108

(c) Georg Thieme Verlag KG Stuttgart · New York

ISSN 0303-4259

Korrespondenzadresse

Dr. Dana Bichescu-Burian, Klinik für Psychiatrie und

Psychotherapie I der Universität Ulm, Zentrum für

Psychiatrie Südwürttemberg, Weingartshofer Straße 2,

88214 Ravensburg

DanaMaria.BichescuBurian@zfp-zentrum.de

\section{ZUSAMMENFASSUNG}

Chronisch verlaufende posttraumatische Belastungsstörungen (PTBS) erschweren die psychosoziale Integration von Flüchtlingen. Wir berichten über eine erfolgreiche narrative Expositionstherapie (NET) bei einem 42-jährigen Flüchtling aus Zentralafrika mit schwerer Psychopathologie, die mit Hilfe des stationären Settings möglich wurde. Der Therapieverlauf unterstreicht die Wirksamkeit und Notwendigkeit der Intervention auch bei komplizierten Fällen.

\section{ABSTRACT}

Forms of Posttraumatic stress disorder (PTSD) with chronical course hamper the psychosocial integration of refugees. We here report the successful treatment according to the approach of Narrative Exposure Therapy (NET) of a severely affected 42-year-old refugee from Central Africa, facilitated by an inpatient care setting. The treatment course underlines the effectiveness and necessity of the intervention even in more complex cases.

\section{Einleitung}

Der geschätzte Anteil von Geflüchteten mit behandlungsbedürftigen psychischen Beschwerden in Deutschland beträgt $50 \%$ [1]. Die PTBS-Rate ist im Vergleich zur Allgemeinbevölkerung um das Zehnfache erhöht [2]. Das bisherige Therapieangebot an spezialisierten Einrichtungen ist dafür bei Weitem nicht ausreichend [3-5].

\section{Fallbericht}

\section{Aufnahmeanlass/Diagnostik}

Wir berichten über einen 42-jährigen Mann aus Nigeria, uns aus der Institutsambulanz zugewiesen bei ausbleibender Besserung unter ambulanter Behandlung mit supportiven Gesprächen,
Antidepressiva und Neuroleptika. Auslöser der akuten Krise war die Ermordung seines Sohnes. In Nigeria war er Folteropfer, Zeuge von Morden und verlor Verwandte durch gewaltsamen Tod. In Deutschland arbeitete er bei einem privaten Bauunternehmer, wurde aber nach der psychischen Destabilisierung gekündigt. Die restliche Familie war in Nigeria. Seine sozialen Kompetenzen waren durch die Symptomatik eingeschränkt, sodass er bei Aufnahme zurückgezogen und ausgegrenzt im Flüchtlingsheim lebte. Seine Flüchtlingshelferin war allerdings sehr unterstützend.

Der Patient zeigte schwerste posttraumatische und dissoziative Symptome, Halluzinationen und Bedrohungserleben sowie Selbstvorwürfe und Trauergefühle. Zudem klagte er zunehmend über somatische Symptome. Psychodiagnostisch lagen 
eine PTBS mit dissoziativen Symptomen und eine schwere depressive Episode vor.

Folgende psychologische Tests wurden eingesetzt: die PTBS Checkliste für DSM-5 (PCL-5) [6], der Fragebogen zu dissoziativen Symptomen (FDS) [7] und die Symptom-Checkliste (SCL90-R) [8]. Trotz deutlichen Rückgangs von der Aufnahme bis zur Entlassung (PCL-5-Summenwert 76 auf 51, FDS Gesamtwert 57,5 auf 13,2 und SCL-90-R-GSI-Wert 2,87 auf 1,11) blieb die Symptomausprägung klinisch relevant.

\section{Therapeutischer Verlauf}

Entscheidend für die Krisenintervention zu Beginn waren die Herausnahme aus dem Alltag, Einzelgespräche sowie die psychopharmakologische Behandlung der Schlafstörungen, Unruhezustände und des psychotischen Erlebens. Nach Psychoedukation konnte der Patient annehmen, dass die Sinnestäuschungen Flashbacks waren.

Die anschließend geplante traumafokussierte Behandlung in einer Universitätsambulanz wurde aufgrund mangelnder Kapazitäten abgesagt. Wir vereinbarten daher die stationäre Durchführung einer solchen Therapie vor dem Hintergrund der rezidivierenden suizidalen Krisen und des dauerhaften Arousals, was die psychosoziale Versorgungsplanung nahezu unmöglich machte.

Die geplante NET zielte v. a. auf die Habituation zu den traumabezogenen Reizen sowie die Integration der Erinnerungen in das autobiografische Gedächtnis [9]. Neben klassischen Elementen wie In-sensu-Exposition und kognitiver Umstrukturierung, enthält die NET auch einige Besonderheiten. Ursprünglich als Kurzintervention für Krisengebiete entwickelt, ist ihre kulturübergreifende Effektivität inzwischen gut belegt. Schwersttraumatische Szenen werden auf allen Ebenen wiedererfahren, positive Erlebnisse dazwischen aktivieren Ressourcen. Zur Ermöglichung der Gedächtnisintegration durch eine raumzeitliche Rückbindung erfolgt die Verarbeitung der traumatischen Szenen im Kontext der kompletten Lebensgeschichte, in chronologischer Reihenfolge. Durch ein Seil und Steine, Blumen, Kerzen und Stöcke wird in der ersten Sitzung eine Lebenslinie mit bedeutsamen Lebensereignissen abgebildet. Während der Exposition platziert sich der Patient in der sicheren Gegenwart, die eine Distanz zum „Damals“ sowie die Benennung der Geschehnisse ermöglicht. Ein Narrativ wird schriftlich erstellt und bei jeder Sitzung erneut durchlebt und weitergeführt. Dies kann zur Menschenrechtsarbeit verwendet werden.

Die insgesamt 10 NET-Sitzungen wurden vorwiegend wöchentlich von einer Psychologischen Psychotherapeutin mit vertiefter Ausbildung in Psychotraumatologie durchgeführt. Aus kostenökonomischen Gründen wurden nur die schwersten Traumata mit jeweils maximal 2 In-sensu-Expositionen verarbeitet. Die Behandlung erfolgte auf Englisch, die guten Sprachfertigkeiten des Patienten erübrigten den Einsatz eines Dolmetschers. Der Dialekt und kulturelle Hintergrund erforderten allerdings den Einsatz von Bildern und bildhafter Sprache um Vergleiche heranzuziehen und traumatische Szenen genauer nachzustellen. Dies entspricht der NET-Arbeitsweise und ermöglicht eine intensivere Exposition. Bei seiner Lebenslinie überwogen die Traumata deutlich, der Patient arbeitete strukturiert und konnte größtenteils eine „Vogelperspektive“ einhalten.

Durch die Bearbeitung der positiven und mäßig belastenden Kindheitserinnerungen zu Beginn der NET erlernte der Patient das Prozedere. Aufgrund der emotionalen Belastung konnte er sich manchmal nur mithilfe von Bedarfsmedikation oder Skills, etwa Aromatherapie, wieder regulieren. Während der Exposition mit Ereignissen, bei denen er Zeuge von Ermordungen war oder gefoltert wurde, war ein erhöhter Einsatz von antidissoziativen Interventionen notwendig [10].

Hinsichtlich des Todes seines Sohnes entstand zunächst eine Trauerblockade mit Schuldgefühlen und Akzeptanzproblemen des Verlusts. Es kam zum stärksten Wiederanstieg von Schlafstörungen und Unruhezuständen. Wut und aggressive Ausbrüche gegen Mitpatienten erforderten die pflegerische und therapeutische Intervention. Laut der NET entstehen solche Emotionen sekundär durch die Traumaverarbeitung. Sie werden Teil des „Furchtnetzwerks“ und provozieren somit traumatische Erinnerungen und flucht- und kampfartige Reaktionen [9]. Während der Gedächtnisaktivierung sieht die NET deshalb auch kognitive Interventionen vor. Dadurch erkannte der Patient, dass er die Geschehnisse nicht beeinflussen hätte können und eher die Täter Schuld oder Scham empfinden sollten. Die vertrauensvolle therapeutische Beziehung im Sinne von NET ermöglichte dem Patienten korrigierende Erfahrungen diesbezüglich. Nach Psychoedukation über Trauerreaktionen und Umgang mit Trauer wurde der Patient unterstützt den Verlust zu akzeptieren und seinen Abschied individuell zu gestalten.

Nachdem eine leichte Stimmungsaufhellung auftrat, erfolgte die Einteilung in eine körperlich nicht anspruchsvolle Arbeitstherapie, die weitestgehend durchgehalten wurde. Die stabilen Phasen wurden länger. Dies spiegelte sich auch in den psychologischen Tests wider. Die Entlassung wurde mit Beurlaubungen zur Außenorientierung vorbereitet. Die weitere Behandlung erfolgt nun durch die PIA und ambulante Ergotherapie.

Bisher kam es zu keiner stationären Wiederaufnahme. Der Patient nahm regelmäßig an Behandlungsangeboten im Rahmen der PIA teil. Es kam zu einer weiteren Besserung des Symptom- und Leistungsbilds, sodass er in naher Zukunft eine Stelle auf dem ersten Arbeitsmarkt anstrebt.

\section{Diskussion}

Der Überlebenskampf trotz schwerster Traumatisierung sowie das erreichte Funktionsniveau in Deutschland deuteten auf psychische Ressourcen des Patienten hin und verbesserten die Prognose bei störungsspezifischer Psychotherapie. Die Symptomzunahme nach der Ermordung des Sohnes machte die stationäre Vorstellung erforderlich. Dies entspricht dem Modell des kumulativen Effekts der Traumata auf die Symptome [11].

Diagnostisch interpretierten wir die kognitiven Einschränkungen und die psychotischen Symptome als dissoziative Psychopathologie. Die soziale Situation kam erschwerend hinzu. Neben Entlastung und Schutz ermöglichte das stationäre Setting eine genauere Einschätzung sowie eine unterstützende Psychopharmakotherapie. Dysfunktionale Verhaltensweisen 
konnten dadurch auch zeitnah festgestellt und bearbeitet werden. Ebenso waren kurzfristige Interventionen zur Emotionsregulation möglich. Das multiprofessionelle Setting ermöglichte auch die adäquate Durchführung somatischer Untersuchungen und Behandlungen in anderen Facheinrichtungen. Dies war bei Wechselwirkung somatischer und psychischer Symptome dringend indiziert.

Es gibt bisher keine wissenschaftlich zuverlässige Erfassung der psychotherapeutischen/psychiatrischen Versorgung von Flüchtlingen in Deutschland [12]. Bemühungen beim Ausbau der Angebote in der Regelversorgung gepaart mit Spezialzentren für die Behandlung traumatisierter Flüchtlinge machen sich zwar bemerkbar $[12,13]$. Es bedarf aber eines weiteren Ausbaus der psychodiagnostischen, psychotherapeutischen und interkulturellen Infrastruktur sowie auch Dolmetsch-, Finanzierungs- und Vernetzungsstrategien [12, 13]. Der Verlauf zeigt, dass durch die NET die weitere psychosoziale Versorgungsplanung erst möglich wurde. Das bisher ausreichende ambulante Setting nach Entlassung werten wir angesichts der rezidivierenden Krisen im Vorfeld der NET als Zeichen für eine anhaltende Stabilisierung. Dies ist aus unserer Sicht ermutigend für die weitere Anpassung der Regelversorgung an die Bedürfnisse dieser Klientel. Vor allem der Ausbau von Kompetenzen für kulturübergreifende, traumafokussierte Kurzzeitinterventionen in der Regelversorgung könnte inadäquate Interventionen und Wartezeiten vermeiden und somit auch Chronifizierung vorbeugen und Ressourcen schonen.

\section{FAZIT}

- Die Durchführung einer NET bei Flüchtlingen ist im Rahmen der stationären Regelversorgung möglich.

- Bei erheblicher Symptombelastung und fehlendem „sicheren Raum“ kann das stationäre Setting sogar als Voraussetzung für die Durchführung der NET gesehen werden.

- Der Rückgang der Symptombelastung durch eine traumafokussierte Behandlung ist eine Voraussetzung für eine weitere psychosoziale Versorgungsplanung und gelingende Integration.

\section{Interessenkonflikt}

Die Autoren geben an, dass kein Interessenkonflikt besteht.

\section{Literatur}

[1] EPA. Position Paper on Psychiatric Care of Refugees in Europe. 2015

[2] Bundespsychotherapeutenkammer (BPtK). Mindestens die Hälfte der Flüchtlinge ist psychisch krank. (16.09.2015). Im Internet: http:// www.bptk.de/aktuell/einzelseite/artikel/mindestens-d.html (Stand: 04.02.2018)

[3] Deutsche Gesellschaft für Psychiatrie und Psychotherapie (DGPPN). Psychosoziale Versorgung von Flüchtlingen verbessern. (22.03.2016). Im Internet: https://www.dgppn.de/_Resources/Persistent/ c03a6dbf7dcdb0a77dbdf4ed3e50981431abe372/2016_03_22_ DGPPN-Positionspapier_psychosoziale\%20Versorgung\%20Fl\%C3\% BCchtlinge.pdf (Stand: 04.02.2018)

[4] Silove D, Ventevogel P, Rees S. The contemporary refugee crisis: an overview of mental health challenges. World Psychiatry 2017; 16: $130-139$

[5] Schneider F, Bajbouj M, Heinz A. Psychische Versorgung von Flüchtlingen in Deutschland. Nervenarzt 2017; 88: 10-17

[6] Schauer M, Neuner F, Elbert T. Narrative exposure therapy: A shortterm treatment for traumatic stress disorders. Cambridge, Göttingen: Hogrefe Publishing; 2011

[7] Schauer M, Elbert T. Dissociation following traumatic stress: Etiology and treatment. Z Psychol 2010; 218: 109

[8] Blevins CA, Weathers FW, Davis MT et al. The Posttraumatic Stress Disorder Checklist for DSM-5 (PCL-5): Development and initial psychometric evaluation. J Trauma Stress 2015; 28: 489-498

[9] Spitzer C, Stieglitz RD, Freyberger HJ. FDS. Fragebogen zu Dissoziativen Symptomen. Göttingen: Hans Huber; 2014

[10] Franke GH, Derogatis LR. Symptom-Checkliste von LR Derogatis: SCL90-R; deutsche Version. Göttingen: Beltz Test; 2002

[11] Mollica RF, McInnes K, Poole C et al. Dose-effect relationships of trauma to symptoms of depression and post-traumatic stress disorder among Cambodian survivors of mass violence. $\mathrm{Br}$ J Psychiatry 1998; $173: 482-488$

[12] Gouzoulis-Mayfrank E, Schmitz-Buhl M, Schaffrath J et al. Die aktuelle Situation der Versorgung von Flüchtlingen in psychiatrischen Kliniken in Deutschland - Eine Bestandsaufnahme der BDK. Psychiat Prax 2017; 44: 7-9

[13] Bender M. Spezialstationen für die psychiatrisch-psychotherapeutische Behandlung von Flüchtlingen - Pro. Psychiat Prax 2016; 43: $131-132$ 